\title{
Role of endoscopic treatment or balloon-occluded retrograde transvenous obliteration in patients with Child-Pugh class $C$ end-stage liver cirrhosis and esophageal/gastric varices
}

\author{
Jin Wook Chung \\ Department of Radiology, Seoul National University Hospital, Seoul National University College of Medicine, Seoul, Korea
}

Keywords: Esophageal and gastric varices; Liver cirrhosis; Hemostasis, endoscopic; Portal hypertension; Radiology, interventional

\section{See Article on Page 183}

Child-Pugh $C$ disease is a strong prognostic indicator of liver diseases, not only in their natural history but also after active treatments for them.'

Conservative or pharmacologic therapy alone frequently fails in patients with Child-Pugh C disease and variceal bleeding. Therefore, it is inevitable to perform invasive treatments, such as endoscopic injection sclerotherapy (EIS) or endoscopic variceal ligation (EVL) or balloon-occluded retrograde transvenous obliteration (BRTO), to stop bleeding or to prevent rebleeding.

In the current issue, Yokoyama et al. conducted a retrospective study to evaluate the safety and prophylactic effect of variceal treatment with endoscopic procedures and BRTO in advanced liver cirrhosis with Child-Pugh C disease. ${ }^{2}$ They included 51 patients with Child-Pugh C end-stage liver cirrhosis who underwent endo- scopic procedures or BRTO for esophageal/gastric varices between April 1995 and December 2017, and analyzed (1) the overall survival rates; (2) comparison of the overall survival rates and changes in Child-Pugh score (CPS) in the endoscopic treatment group $(n=39)$ and the BRTO group $(n=12)$; and, (3) factors contributing to death within 1 year of treatment. They reported favorable survival outcome of the patients $(72.6 \%, 50.1 \%, 30.2 \%$ and $15.1 \%$ at 1, 2, 3 and 5 years, respectively) without significant difference between endoscopic treatments and BRTO. The average of CPS from before treatment to one month after variceal treatment significantly improved from 10.53 to $10.02(P=0.003)$. Three significant factors that contributed to death within one year after treatment were identified, including the presence of bleeding varices, high CPS ( $\geq 11)$, and high serum total bilirubin levels ( $\geq 4.0 \mathrm{mg} / \mathrm{dL}$ ).

In comparing overall survival with historical controls, selection bias for a specific treatment and recent improvements in general patient management should be considered. Even though the au-

\section{Abbreviations:}

BRTO, balloon-occluded retrograde variceal obliteration; CPS, Child-Pugh score: EIS, endoscopic injection sclerotherapy; EVL, endoscopic variceal ligation

\author{
Corresponding author : Jin Wook Chung \\ Department of Radiology, Seoul National University Hospital, Seoul \\ National University College of Medicine, 101 Daehak-ro, Jongno-gu, \\ Seoul 03080, Korea \\ Tel: +82-2-2072-2584, Fax: +82-2-743-6385 \\ E-mail: chungjw@snu.ac.kr \\ https://orcid.org/0000-0002-1090-6872
}


thors described that a reason for the better survival outcome may be associated with the relatively large proportion of patients with a CPS of 10 in this study, they did not specifically describe the clinical situation when they attempted or avoided invasive treatments. Readers want to know whether the authors included the patients with main portal vein occlusion with large gastrorenal shunt, advanced hepatocellular carcinomas or intractable ascites. Although subgroup analysis may be limited due to sample size, if those groups of patients were included, survival outcome of each group should be reported.

It has been well known that BRTO improves liver function after the procedure by increasing hepatopetal portal flow. ${ }^{3-5}$ However, not every patient shows improved liver function. ${ }^{6}$ Some patients may undergo acute exacerbation of liver function due to rapid increase in portal pressure after BRTO. The authors should analyze and report mortality of the invasive procedures and its prognostic factors. That information will be valuable for readers in selecting optimal candidate of invasive procedures and understanding the changes in liver function after the procedures for Child-Pugh C disease with gastroesophageal varices. Current western guidelines recommend EVL for acute esophageal varix bleeding, EIS for gastric varix bleeding, and EIS could be considered for rescue therapy for esophageal varix bleeding. ${ }^{7}$ The authors merged different treatment methods (EVL and EIS) with different indication (esophageal varix and gastric varix) into a single treatment group, which needs to be considered when the readers interpret this data.

Given that beta-blocker use with EVL is one of important factors associated with survival or change of portal pressure, information of numbers of patients treated with beta-blocker and changes of heart rates by beta-blockers are required to interpret the association between EVL/EIS and survival/safety outcomes in this study. Furthermore, $39.2 \%$ of total population received endoscopic procedures or BRTO for the purpose of prophylactic treatment. Number of patients who achieved eradication of esophageal/gastric varices by endoscopic procedures are helpful to understand the results of liver function changes in this study: just one-time procedure for all of study periods or not. It may be difficult to understand that liver function was improved after prophylactic EVL or EIS procedures (just one time or many) in this study. "Best supportive care" other than EVL/EIS such as antibiotics, hepatotonics, branched amino-acid, or rifaximin treatment can af- fect change of liver function in this study, Those factors should be considered in the multivariable analyses. Recently, western guidelines include early transjugular intrahepatic portosystemic shunt procedure within 72 hours from endoscopic treatments as a treatment option for patients at high risk of failure or rebleeding (Child-Pugh class C cirrhosis or Child-Pugh class B with active bleeding on endoscopy). ${ }^{7}$ Its applicability in Asian countries needs to be evaluated in the future.

\section{Conflicts of Interest}

The author has no conflicts to disclose.

\section{REFERENCES}

1. D'Amico G, Garcia-Tsao G, Pagliaro L. Natural history and prognostic indicators of survival in cirrhosis: a systematic review of 118 studies. J Hepatol 2006;44:217-231.

2. Yokoyama K, Yamauchi R, Shibata K, Fukuda H, Kunimoto H, Takata $\mathrm{K}$, et al. Endoscopic treatment or balloon-occluded retrograde transvenous obliteration is safe for patients with esophageal/gastric varices in Child-Pugh class C end-stage liver cirrhosis. Clin Mol Hepatol 2019;25:183-189.

3. Chikamori F, Kuniyoshi N, Shibuya S, Takase Y. Short-term hemodynamic effects of transjugular retrograde obliteration of gastric varices with gastrorenal shunt. Dig Surg 2000;17:332-336.

4. Miyamoto Y, Oho K, Kumamoto M, Toyonaga A, Sata M. Balloonoccluded retrograde transvenous obliteration improves liver function in patients with cirrhosis and portal hypertension. J Gastroenterol Hepatol 2003;18:934-942.

5. Saad WE, Wagner CC, Al-Osaimi A, Bliebel W, Lippert A, Davies $M G$, et al. The effect of balloon-occluded transvenous obliteration of gastric varices and gastrorenal shunts on the hepatic synthetic function: a comparison between Child-Pugh and model for endstage liver disease scores. Vasc Endovascular Surg 2013;47:281-287.

6. Yamamoto A, Nishida N, Morikawa H, Jogo A, Kageyama K, Sohgawa $E$, et al. Prediction for improvement of liver function after balloon-occluded retrograde transvenous obliteration for gastric varices to manage portosystemic shunt syndrome. J Vasc Interv Radiol 2016;27:1160-1167.

7. Garcia-Tsao G, Abraldes JG, Berzigotti A, Bosch J. Portal hypertensive bleeding in cirrhosis: risk stratification, diagnosis, and management: 2016 practice guidance by the American Association for the study of liver diseases. Hepatology 2017;65:310-335. 